Voix et Images

\title{
Ludisme et clichés dans l'Avalée des avalés de Réjean Ducharme
}

\section{Kenneth W. Meadwell}

Volume 14, numéro 2 (41), hiver 1989

L’édition littéraire au Québec

URI : https://id.erudit.org/iderudit/200776ar

DOI : https://doi.org/10.7202/200776ar

Aller au sommaire du numéro

Éditeur(s)

Université du Québec à Montréal

\section{ISSN}

0318-9201 (imprimé)

1705-933X (numérique)

Découvrir la revue

Citer cet article

Meadwell, K. W. (1989). Ludisme et clichés dans l'Avalée des avalés de Réjean

Ducharme. Voix et Images, 14(2), 294-300. https://doi.org/10.7202/200776ar d'utilisation que vous pouvez consulter en ligne.

https://apropos.erudit.org/fr/usagers/politique-dutilisation/ 


\title{
Ludisme et clichés dans l'Avalée des avalés de Réjean Ducharme
}

\author{
par Kenneth W. Meadwell, Université de Winnipeg
}

La parution en 1966 de l'Avalée des avalés - le premier roman de Réjean Ducharme - représente une étape décisive dans l'évolution des lettres québécoises, cet ouvrage ayant provoqué une remise en cause des formes tant romanesques que critiques qui dominaient à l'époque. Depuis un certain nombre d'années, la critique thématique et socio-historique cohabite avec une approche plus immanente du texte en tant que code limitatif et prescriptif ${ }^{1}$ d'après la $^{-1}$ terminologie riffaterrienne. Spécificité esthétique, littérarité, unicité du texte sont autant de notions communément invoquées dans cette optique. Déceler la fonction et le fonctionnement de l'activité scripturale chez Ducharme équivaut à mettre en évidence les caractéristiques qui font de l'Avalée des avalés la partie intégrante d'un ensemble plus vaste, constant dans sa nature, mais variable dans ses apparences. Recourir à la réalité extra-linguistique en vue d'étudier l'élément littéraire chez Ducharme ne peut qu'aboutir à des analyses dont la pertinence littéraire serait quelque peu limitée. Il ne serait pas inutile à ce sujet de rappeler la formule de Hegel: [...] quand l'art s'en tient au but formel de la stricte imitation, il ne nous donne, à la place du réel et du vivant, que la caricature de la vie. ${ }^{2} \mathrm{Au}$ dire de Michael Riffaterre, la création de l'univers immanent de l'œuvre littéraire est régie au niveau syntagmatique:

Il n' est question que de constater comment les phrases, à mesure qu'on les déchiffre, semblent engendrées de manière nécessaire; de constater comment l'énoncé, loin de se modeler sur un modèle non verbal, se plie aux impératifs d'associations sémantiques et formelles entre les mots. Associations qui limitent étroitement les options offertes, pour chaque point de l'énoncé, au déroulement ultérieur de la phrase. ${ }^{3}$

Dans l'Avalée des avalés, la sémiosis l'emporte sur la mimésis, et ce, justement par le renversement ludique de l'image stéréotypée de l'enfant.

Dès l'ouverture du réçit, Bérénice Einberg se présente comme un être fort singulier, de sorte que le lecteur, se sentant sur un terrain incertain, s'efforce de concilier ce que dit la narratrice avec ses propres codes du «naturel» ou du «vraisemblable».

1 Michael Riffaterre, la Production du texte, Paris, Seuil, 1979, p. 11 (Poétique).

2 Georg Hegel, Textes choisis, Paris, PUF, 1981, p. 13.

3 Riffaterre, op. cit., p. 179. 
Je suis seule et j' ai peur. Quand j' ai faim, je mange des pissenlits par la racine et ça se passe. Quand j'ai soif, je plonge mon visage dans l'un des seaux et j'aspire. Mes cheveux déboulent dans l'eau. J'aspire et ça se passe: je n' ai plus soif, c'est comme si je n'avais jamais eu soif. ${ }^{4}$

Solitude et peur, deux thèmes récurrents, s'unissent dans le portrait de Bérénice qui, pour sauvegarder son autonomie et son innocence d'enfant, cultive une lucidité quelque peu extravagante et toujours susceptible d'étonner le lecteur:

Quand je ne suis pas seule, je me sens malade, en danger. J'ai ma peur à vaincre. Pour vaincre la peur, il faut la voir, l'entendre, la sentir. Pour voir la peur, il faut être seul avec elle. Quand je perds ma peur de vue, c'est comme si je perdais connaissance. C'est peut-être parce que j'ai été sevrée deux jours après ma naissance. Ce sont eux qui m'ont sevrée. Mais j'aime mieux croire que je me suis sevrée moi-même, que, dans un grand élan d'orgueil, $j$ 'ai mordu le sein de ma mère, que j'avais des dents de fer rouillé et que le sein s'est gangrené. J' imagine toutes sortes de choses et je les crois, je les fais agir sur moi comme si elles étaient vraies. Il n'y a de vrai, que ce que j' ose croire vrai. (p. 15-16)

L'hésitation première du lecteur provient non pas du statut incertain de l'événement évoqué, événement qui n'a évidemment pas eu lieu, mais du simple fait que la narratrice l'invente. En somme, c'est Bérénice elle-même, de par sa nature invraisemblable, qui motive l'étonnement engendré par la violence inattendue de ses propos:

Il est l'heure que je me mette à tuer des hommes blancs, des femmes blanches et des enfants blancs avec un tisonnier. Demain, il sera trop tard. L' heure de broyer des mains et des pieds avec des étaux lents et de recueillir le sang exprimé dans une choppe sonne. Boire du sang. C'est si chaud du sang. C'est comme du lait au sortir de la vache. (p. 219)

Ce passage n'est pas sans rappeler le fantastique ténébreux ${ }^{5}$ chez Lautréamont où l'on peut lire sur un ton tout aussi macabre, mais d'autant plus répugnant à cause de l'évocation des larmes de la victime:

On doit laisser pousser ses ongles pendant quinze jours. Oh! comme il est doux d'arracher brutalement de son lit un enfant qui n'a rien encore sur la lèvre supérieure, et, avec les yeux très ouverts, de faire semblant de passer suavement la main sur son front, en inclinant en arrière ses beaux cheveux! Puis, tout à coup, au moment où il s'attend le moins, d'enfoncer les ongles longs dans sa poitrine molle, de façon qu'il ne meure pas; car, s'il mourait, on n'aurait pas plus tard l'aspect

4 Réjean Ducharme, l'Avalée des avalés, Paris, Gallimard, 1966, p. 7-8. Toute référence ultéricure à cet ouvrage sera suivie uniquement du numéro de page entre parenthèses.

5 Raymond Jean, Lectures du désir, Paris, Seuil, 1977, p. 104 (Points). 
de ses misères. Ensuite, on boit le sang en léchant les blessures; et, pendant ce temps, qui devrait durer autant que l'éternité dure, l'enfant pleure. Rien n'est si bon que son sang, extrait comme je viens de le dire, et tout chaud encore, si ce ne sont ses larmes, amères comme le sel. 6

L'une des distinctions à faire entre le passage tiré de l'Avalée des avalés et celui provenant des Chants de Maldoror réside dans la réaction suscitée chez le lecteur, car bien que partageant quelques structures thématiques identiques, les deux textes motivent une lecture différente.

Il est permis à Bérénice Einberg, par le biais de l'individuation «enfant», de proposer un discours tout aussi imprégné de violence et de haine; celui du narrateur des Chants de Maldoror ne produit cependant pas le même effet de répulsion chez le lecteur. Si ce dernier se laisse convaincre, n'est-ce pas dû précisément à l'actualisation implicite d'un lieu commun dans la narration selon lequel l'enfant référentiel devient prétexte à l'invention, tant du côté syntaxique que sémantique. Aussi le lecteur profite de l'image stéréotypée de l'enfant référentiel pour adoucir sa répugnance, alors qu'a priori, la force est la même que chez Lautréamont, le narrateur ayant ici atteint l'âge de raison. On voit donc, quoique les deux voix narratives se définissent par les mêmes procédures de mise en discours, que les messages ultimes n'en sont pas moins décodés de façons différentes.

Le statut d'enfant accorde à la narratrice ducharmienne une grande latitude fictionnelle, car on reconnaît dans son discours l'existence de quelques clichés, qui ont néanmoins subi une certaine dislocation, et qui permettent au lecteur d'accepter l'irréalité du discours comme «normale» pour ainsi dire. En revanche, le narrateur revêt chez Lautréamont une forme si hideuse et si loin de tout stéréotype qu'il est incapable de reproduire l'élément ludique que l'on trouve chez Bérénice. Le narrateur se présente ainsi:

Je suis le fils de l'homme et de la femme. [...] Moi, si cela avait pu dépendre de ma volonté, j'aurais voulu être plutôt le fils de la femelle du requin, dont la faim est amie des tempêtes, et du tigre, à la cruauté reconnue. [...] Vous, qui me regardez, éloignez-vous de moi, car mon haleine exhale un souffle empoisonné. Nul n'a encore vu les rides vertes de mon front; ni les os en saillie de ma figure maigre, pareils aux arêtes de quelque grand poisson, ou aux rochers couvrant les rivages de la mer, ou aux abruptes montagnes alpestres, que je parcourus souvent, quand $j$ ' avais sur ma tête des cheveux d' une autre couleur. Et, quand je rôde autour des habitations des hommes, pendant les nuits orageuses, les yeux ardents, les cheveux flagelés par le vent des tempêtes, isolé comme une pierre au milieu du chemin, je couvre ma face flétrie, avec un morceau de velours, noir comme la

6 Lautréamont et Germain Nouveau, CEuvres complètes, Paris, NRF/Gallimard, 1970 , p. 49 (Bibliothèque de la Pléiade). 
suie qui remplit l'intérieur des cheminées: il ne faut pas que les yeux soient témoins de la laideur que l'Être suprême, avec un sourire de haine puissante, a mise sur moi. ${ }^{7}$

L'autoportrait de Bérénice, si désobligeant soit-il, réunit paradoxalement trois éléments que l'on associe normalement au nouveau-né, à l'enfant et à l'adolescent:

J'ai le visage tissé de boutons. Je suis laide comme un cendrier rempli de restes de cigares et de cigarettes. Plus il fait chaud, plus mes boutons me font mal. J'ai le visage rouge et jaune, comme si j'avais à la fois la jaunisse et la rougeole. Mon visage durcit, épaissit, brûle. (p. 16)

Aussi constate-t-on que Bérénice est «née» de la transformation de trois images stéréotypées: nouveau-né ictérique, enfant souffrant de la rougeole et adolescent boutonneux. Cet être romanesque, aussi peu vraisemblable que le narrateur des Chants de Maldoror, est cependant inspiré du tableau humain de la création qui se dessine à partir d'une comparaison extraordinaire entre os, arêtes de poisson, rochers et montagnes. La narratrice semble ironiser sur la peinture qu'elle fait d'elle-même par le truchement d'une comparaison non conventionnelle chez Ducharme: Je suis laide comme un cendrier rempli de restes de cigares et de cigarettes. Force est de noter que le ludisme ainsi manifesté chez Ducharme est entièrement absent du texte de Lautréamont.

Chez Ducharme, le glissement de l'abstrait au concret ${ }^{8}$ - «laideur» comparée à «cendrier» - par la dislocation humoristique du syntagme «être laid comme...», provoque le lecteur à cause de l'inattendu de cette modification. Celui-ci ne peut qu'être sensible au ludisme ducharmien, ce qui joue, par conséquent, un rôle déterminant dans la manière dont il réagit au texte.

Une série de citations anaphoriques permet de cerner le processus par lequel le cliché ainsi modifié ne se caractérise plus par la causalité conventionnelle à laquelle tout lecteur s'attend. Les trois citations suivantes offrent des réponses éventuelles à la question (non posée explicitement dans le texte) de l'adulte à l'enfant: «Que voudrais-tu faire quand tu seras grande?» C'est la narratrice qui parle:

Quand je serai grande, je serai arrogante et impie. J'aurai poussé des racines grosses comme les colonnes de la synagogue. J'aurai des feuilles grandes comme des voiles. Je marcherai tête haute. Je ne verrai personne. Quand le feu qui vient viendra, il brûlera ma peau, mais mes os ne flancheront pas, mais mon échine ne fléchira pas. (p. 18)

Quand je serai grande, je n' aurai plus en place de caur qu' une outre vide et sèche. Christian me laissera froide, tout à fait indifférente. Aucun lien ne nous unira que je n'aurai tissé de mes propres mains.

7 Lautréamont, ibid., p. 54-55.

8 À ce propos, voir Patrick Imbert, Roman québécois contemporain et clichés, Ottawa, Presses de l'Université d'Ottawa, 1983, p. 116-118. 
Aucun élan ne me portera vers lui de mes seuls pieds. J'aimerai sans amour, sans souffrir comme si j'étais quartz. Je vivrai sans que mon cour batte, sans avoir de cœur. (p. 30)

Quand je serai grande, je ne passerai pas mon temps à déambuler paresseusement dans l' herbe morte. Je serai donc partie pour un lieu $d^{\prime}$ où on ne revient pas, un lieu où l'on arrive en passant par des lieux où l'on ne s'arrête pas. Je monterai Pégase, comme Ajax d'Ö̈lée, comme Bellérophon. Je mourrai en pleine force, de l'explosion même de ma violence. Je me mesurerai à la mort en plein midi, plein éveil, pleine gloire. (p. 120)

Les trois passages ci-dessus rendent prévisible une structure s'annonçant de prime abord comme celle du cliché prononcé par l'enfant: «Quand je serai grande, je voudrais...» Cela s'annule par un énoncé que caractérisent la figuration de l'adynaton ainsi que l'expression d'une volonté de puissance hyperbolique. Chaque citation souligne un trait de la narratrice, respectivement dans les trois passages: air de supériorité, absence de toute émotion et, enfin, aspiration vers la lucidité dans la mort comme on l'a vu dans l'anticipation de la mort chez Lautréamont. Les allusions à Pégase - symbole de l'inspiration poétique ainsi qu'à Ajax - héros homérique et roi de Locriens - et à Bellérophon — dompteur de Pégase - accentuent le côté extraordinaire de ces comparaisons et font entrevoir chez Ducharme la mise en cause du procédé mimétique.

Une autre manière de subvertir le stéréotype de l'enfant se révèle lors de la création du «bérénicien» par la narratrice, langue à elle qui, malgré ses inventions tant sémantiques que syntaxiques, est loin de revêtir la forme du babil enfantin. Patrick Imbert a décelé chez Ducharme cette imprévisibilité qui va à l'encontre des habitudes langagières les plus fortement établies. ${ }^{9}$ De même, Jacqueline Gerols relève dans l'Avalée des avalés une remise en question du langage ${ }^{10}$, et refuse, à l'instar d'Imbert, d'y voir un apport direct à la production du texte. Certes, néologismes et jeux phonétiques éveillent l'intérêt du lectcur, mais ils jouent aussi un rôle important dans l'orientation de la lecture. L'intérêt principal du bérénicien ne réside pas entièrement dans les manifestations de cet autre langage; dans son fonctionnement, il renverse et réinvente le licu commun qu'est la création d'une langue par l'enfant.

Le bérénicien est né non du désir de communiquer, mais du besoin impérieux que ressent Bérénice de se retirer du monde de l'adulte. Il est donc paradoxal que la narratrice se serve du langage pour se distancer d'autrui, là où l'enfant référentiel pratique son babil enfantin pour se rapprocher des autres. Conséquente dans ses démarches, Bérénice Einberg choisit la réalité linguistique ou extra-linguistique qu'elle vit:

9 Patrick Imbert, «Révolution culturelle et clichés chez Réjean Ducharme», Journal of Canadian Fiction, nos 25-26, 1979, p. 227.

10 Jacqueline Gcrols, «L'invention verbale chez Réjean Ducharme», thèse de maîtrise, Montréal, Université de Montréal, 1970, p. 47. 
Je donne arbitrairement une autre forme à toutes choses qui, par son manque de consistance ou par son immensité, est impossible à saisir... et alors, à la faveur de cette autre forme, je saisis la chose, je la prends dans mes mains, dans mes bras, mais surtout: dans ma tête... (p. 153)

Ainsi est inventé le bérénicien:

Je hais tellement l'adulte, le renie avec tant de colère, que j'ai dî jeter les fondements d' une nouvelle langue... Frappée de génie, devenue ectoplasme, je criai, mordant dans chaque syllabe: «Spétermatorinx étanglobe!»... Le bérénicien comporte plusieurs synonymes. «Mounonstre béxéroorisiduel» et »spétermatorinx étanglobe» sont synonymes. En bérénicien, le verbe être ne se conjugue pas sans le verbe avoir. (p. 250)

Il est intéressant de constater que l'apport proprement sémantique du bérénicien à la trame du récit est à peu près nul; cette nouvelle langue n'est évoquée que dans un seul paragraphe. Le sens de ces expressions inventées demeure indéterminé; cependant, ne possèdent-elles pas une signification évidente puisque leur microcontexte régit leur motivation? Ainsi, le lecteur perçoit que les syntagmes «spétermatorinx étanglobe» et «mounonstre béxéroorisiduel» se posent en réaction au monde des adultes. Ce dernier est non seulement décrit en termes peu flatteurs, il est de plus un référent dans une langue dont le sens n'est accessible qu'à l'enfant.

On voit donc que, s'étant fondée sur le renversement de l'image stéréotypée de l'enfant essayant de se rapprocher de l'adulte en communiquant avec celui-ci grâce à son babil enfantin, la création de la narratrice/enfant dans l'Avalée des avalés entraîne une certaine imprévisibilité au niveau de l'acteur. Ce manque de vraisemblance oriente le lecteur; sa réaction, après l'étape de l'hésitation, est d'accepter sans répugnance le discours de la narratrice. Et ce, justement parce que son discours est loin d'évoquer le malaise que l'on éprouve en lisant les Chants de Maldoror. En privilégiant le ludisme et l'ironie humoristique de Bérénice, le lecteur est justifié, voire amusé, par un tel discours. Il est important de remarquer par ailleurs que même dans son ludisme, Bérénice Einberg subvertit la stéréotypie de l'enfant. Un exemple suffira à illustrer mon propos: arrêtés à un moment donné par la police, lors d'une «évasion» de la demeure familiale, Christian et sa sœur se trouvent encore une fois sous la domination de l'adulte. Quand on leur demande leur adresse, Bérénice répond: Notre adresse, messieurs, c'est: Monsieur et Madame Homme, Planète Terre, Système solaire, Infini. Ôtez donc vos chapeaux, goujats! (p. 118)11. Bérénice modifie non seulement le jeu des jeunes écoliers qui écrivent dans leur cahier leur adresse en

11 Cette citation rappelle ce qu'écrit Stephen Dedalus, protagoniste principal de A Portrait of the Artist as a Young Man de James Joyce (New York, Viking Press, 1982, p. 15), dans un manuel de géographie: Stephen Dedalus / Class of Elements / Clongowes Wood College / Sallins / County / Kildare I Ireland / Europe / The World / The Universe. 
donnant nom de rue, ville, province, pays, continent, hémisphère, planète et ainsi de suite, mais encore elle affiche une attitude irrespectueuse envers les policiers. L'imprécision dans l'adresse qu'elle propose en omettant les données essentielles ainsi que l'épithète destinée aux policiers révèlent nettement qu'elle échappe à la «norme» de l'enfant référentiel et devient l'hyperbole intertextuelle de Stephen Dedalus, «enfant de papier». Elle ne se plie ni aux règles des jeux enfantins ni à la convention sociale selon laquelle l'enfant s'émerveille devant l'uniforme du policier ou du pompier. Il devient donc évident que cette subversion de la stéréotypie de l'enfant, telle qu'on la voit dans le portrait de la narratrice, a pour effet d'orienter la réception du texte vers une acceptation tantôt amusée, car l'ironie est reconnue, tantôt étonnée par des propos sans contraintes. Il est maintenant d'une importance capitale de reconnaitre que les éléments permettant au lecteur d'adopter une attitude diamétralement opposée au malaise éprouvé en lisant Lautréamont sont tous inspirés de la stéréotypie de l'enfant. Et pourtant, on a vu que le texte ne s'approprie nullement cette stéréotypie telle quelle; il l'adapte à ses propres fins, ce qui permet d'accéder à une vision critique du cliché au travers de l'ironie, et plus précisément de l'image dite normale de l'enfant référentiel.

En somme, si devant le personnage de Bérénice, le lecteur ne réagit pas de la même façon que devant le personnage des Chants de Maldoror, ceci est dû aux moyens par lesquels la narratrice est représentée: au niveau descriptif, portrait composé d'images de nouveau-né, d'enfant et d'adolescent métonymiques; au niveau discursif, modification inattendue et amusante de clichés. Il est donc évident que Bérénice Einberg est une enfant «littéraire», une structure hypcrbolique dont la figuration dans l'Avalée des avalés met en cause le bienfondé du cliché, et en particulier tout ce que l'on associe de manière conventionnelle à l'enfant référentiel.

Ce qu'il y a de remarquable dans l'Avalée des avalés, c'est justement ce glissement progressif vers un discours irréel fondé sur une mise en cause du cliché, ouverture qui opère dans le récit tant au niveau de la création du personnage qu'au niveau de l'énonciation. Il est intéressant de remarquer que la fonction signifiante de l'irréel existe à cause de la transformation d'un stérćotype par des instances discursives. Cette image stéréotypée est actualisée et c'est bien ce qui remet en cause le principe mimétique de l'illusion référentielle. Cette illusion extrême est peut-être l'une des meilleures définitions de la littérature ou — plus précisément - de la littérarité. 